Case Report

\title{
A Rare Case of Continuous Type Splenogonadal Fusion in a Young Male with Primary Infertility
}

\author{
Santosh Kumar, Kumar Jayant, Swati Agrawal, \\ Kalpesh Mahesh Parmar, and Shrawan Kumar Singh \\ Department of Urology, PGIMER, Chandigarh 160012, India \\ Correspondence should be addressed to Santosh Kumar; santoshsp1967jaimatadi@yahoo.co.in
}

Received 7 April 2014; Accepted 6 May 2014; Published 14 May 2014

Academic Editor: Elijah O. Kehinde

Copyright (C) 2014 Santosh Kumar et al. This is an open access article distributed under the Creative Commons Attribution License, which permits unrestricted use, distribution, and reproduction in any medium, provided the original work is properly cited.

\begin{abstract}
Splenogonadal fusion is a rare developmental anomaly in which an abnormal connection between splenic tissue and gonads or mesonephric derivatives is present. Here we present a case of young man with the complaint of primary infertility for 3 years. On evaluation (USG and MRI abdomen and pelvis), his right scrotal testis was atrophied and left intra-abdominal undescended testis. On laparoscopic assessment, a mass was seen on the left side due to continuous type of splenogonadal fusion for which excision and left orchidectomy were done. Postoperative period was uneventful and he was discharged under satisfactory condition. Splenogonadal fusion is a rare entity and it is commonly mistaken for testicular tumour. It should be considered in the differential diagnosis of testicular masses especially when there are associated congenital anomalies and preoperative laparoscopic assessment, should be done to avoid unnecessary radical surgery.
\end{abstract}

\section{Introduction}

Splenogonadal fusion is a very uncommon developmental anomaly arising due to abnormal connection between developing splenic tissue from dorsal mesogastrium and gonadal or mesonephric derivatives. Such rare anomaly usually takes place during the 5th to 8th week of gestation, that is, before gonadal descent starts and presents as mass. The product fusion commonly diagnosed as a testicular mass of unknown origin in association with other congenital deformities [1].

\section{Case Report}

A 25-year-old male patient presented in urology clinic with chief complaint of primary infertility of a 3-year duration. General physical examination was normal. On local examination, patient's right atrophic testis was situated in scrotum and of size $1.5 \mathrm{~cm} \times 1.5 \mathrm{~cm}$. On the other hand, there was empty scrotum on left side which was suggestive of undescended testes. There were no associated congenital anomalies seen. Routine blood investigations, renal function test, and liver function tests were normal. The levels of tumor markers as AFP and beta-hCG were $5 \mathrm{kIU} / \mathrm{L}$ and $2.5 \mathrm{kIU} / \mathrm{L}$ which were within normal limits. Semen analysis revealed azoospermia. Hormonal study revealed primary testicular failure with elevated gonadotropin levels. Ultrasonography (USG) revealed absence of left sided testis and epididymis in the scrotum and inguinal canal. MRI reported two oblong masses each $2.5 \times 1 \mathrm{~cm}$ in the left retroperitoneal region anterior and lateral to psoas muscle at L3 to S1 level with atrophied left seminal vesicles (Figure 1). Patient was taken for laparoscopic assessment and was found to have left sided testicular mass with no lymph nodal enlargement (Figure 2). On careful examination, it was suggestive for splenogonadal fusion anomaly for which he underwent left orchidectomy (Figure 3). The specimen was sent for histopathology which showed the tumor mass to be composed of fibrous capsule with red and white pulp within it suggestive of spleen and there was no evidence of spermatogenesis in the testicular portion of resected specimen (Figure 4). He fared well in 


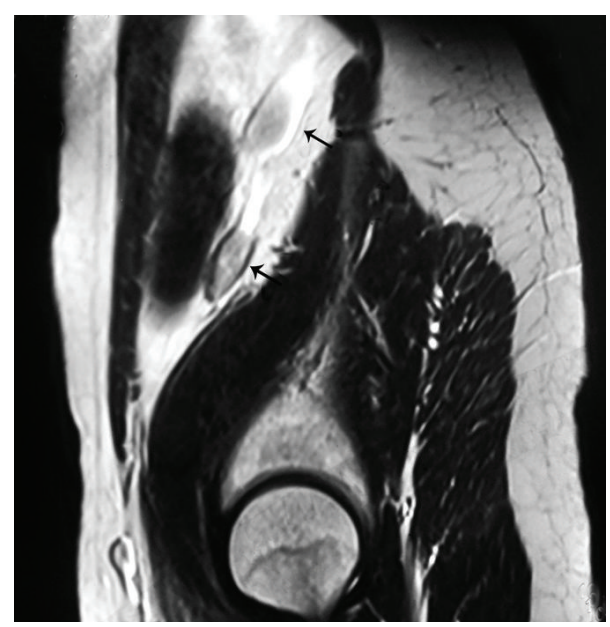

FIGURE 1: MRI T2, sagittal cuts showing 2 oblong masses in the left retroperitoneum.

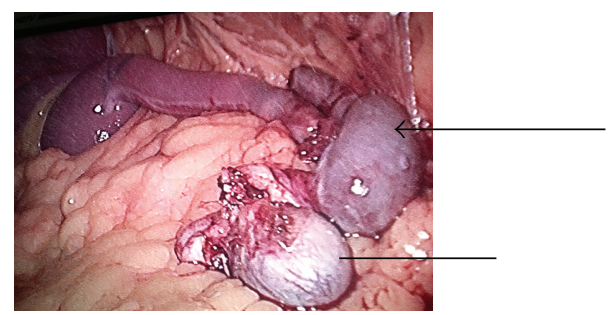

FIGURE 2: Laparoscopic assessment picture showing splenogonadal fusion (splenic portion marked with arrow-line and testicular portion marked with line only).

postoperative period and was discharged on postoperative day 3. Later, he was followed up on out-patient basis and was doing well.

\section{Discussion}

The first description of splenogonadal fusion was made by Bostroem in 1883, and a detailed report by Pommer followed in 1889. There are few other names existing for this disorder in literature as ectopic scrotal spleen or testicular splenic fusion. In a review of 30 cases, Putschar and Manion categorised splenogonadal fusion into continuous and discontinuous types depending on the anatomical continuity between the principal spleen and the gonad [2]. The continuous type is characterized by connection of the spleen and the gonad by a cord of splenic or fibrous tissue. Rarely, beads of splenic tissue are interspersed throughout the fibrous cord. In the discontinuous type, ectopic splenic tissue is attached to the gonad but has no connection to the normally located spleen [3]. Accessory spleen is usually found within the tunica vaginalis and is closely attached to the gonad, although a distinct capsule is present. Splenogonadal fusion is assumed to occur between 5 and 8 weeks of gestation, before the beginning of gonadal descent. Its cause remains unclear, but two theories predominate. First, slight inflammation of the peritoneal surfaces over the spleen and gonadal ridge can

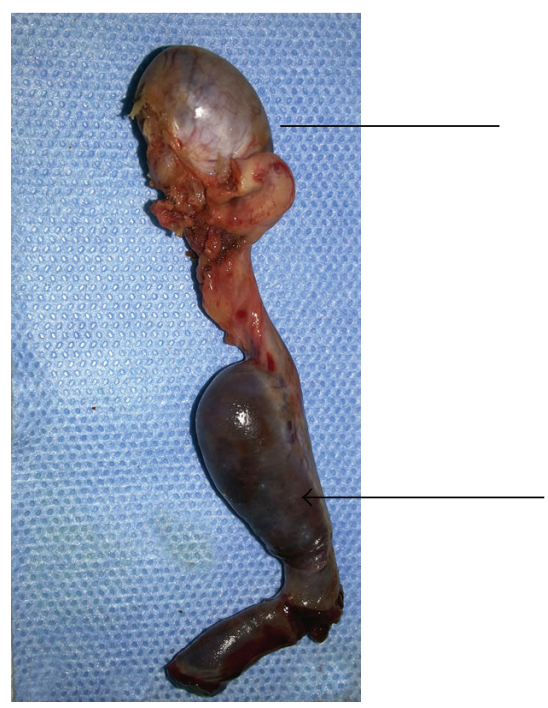

FIGURE 3: Resected specimen of the left orchidectomy with fused splenic tissue (splenic portion marked with arrow-line and testicular portion marked with line only).

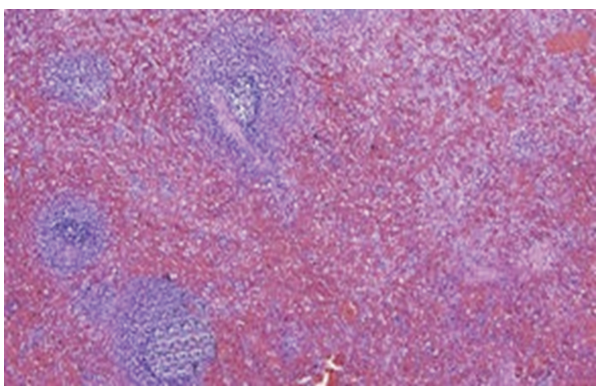

FIGURE 4: Microscopic picture confirming spleen showing white and red pulp in mass. (H\&E stain $\times 400$ magnification).

produce partial fusion of the two organs, while the second has postulated that a retroperitoneal pathway for splenic anlage cells may allow contact with the gonadal anlage [4]. This malformation has predominantly been reported in males, with a male to female ratio of $15: 1$. Most cases are usually seen before the age of 20 and more than half of them were seen before the age of 10 years. Although the left testis is predominantly involved, cases of right splenogonadal fusion have also been reported [5]. Splenogonadal fusion is so rare that it almost never gives any impression of its existence in the nature and thus is rarely diagnosed preoperatively. Mostly, it is incidentally diagnosed as an asymptomatic mass while exploring inguinal region for some other reasons as for cryptorchidism, a hernia, or a hydrocele. Presentation is varied from scrotal mass to testicular torsion, most common presentation being testicular swelling. Another important presentation is acute onset painful scrotal lump secondary to testicular torsion or involvement of the ectopic splenic tissue as a corollary to varied presentation of disorders as in malaria, leukemia, infectious mononucleosis, and traumatic rupture of the ectopic spleen. 
Continuous type of splenogonadal fusion has been associated with many anomalies, including cryptorchidism, which has been found in approximately $33 \%$ of the cases of splenogonadal fusion. Other associated anomalies include cardiac defects, limb bud abnormalities also called peromelia (severe congenital deformity of limbs which closely mimics that of thalidomide embryopathy), micrognathia, hypoglossia, craniosynostosis, spina bifida, palatine, and anorectal and rare syndromic anomalies. Discontinuous type is usually not related to any congenital anomaly though few cases of associated cardiac defects are reported in the literature. Although the case we presented here was of continuous type of splenogonadal fusion, there was no other associated congenital anomaly, except cryptorchidism and infertility [6].

Techniques of diagnostic imaging are available if there is a clinical suspicion of splenogonadal fusion. The most reliable investigation to confirm its diagnosis is preoperative imaging through $99 \mathrm{~m}$ Tc-sulfur colloid liver spleen scan, which detects accessory spleen. Recently, Doppler ultrasonography of the scrotum of patients with a palpable testicular mass has been used to diagnose preoperatively few cases of splenogonadal fusion by visualizing a hypervascular mass on the upper pole of testis and comparing it with patient's own normally located spleen $[7,8]$. Such use of this modality again depends on high index of suspicion in cases of splenogonadal fusion presenting as cryptorchidism, but it may be of value in patients presenting with acute scrotal lump. Unaware of the nature of the left scrotal masses, many surgeons have sacrificed an intact testis because they lacked a proper preoperative diagnosis. Surgeons ignorant of the nature of the scrotal mass and fearing malignant degeneration have performed radical orchidectomy on salvageable testes [9]. In our cases, as the patient had primary gonadal failure and laparoscopic assessment was suggestive of benign testicular mass due to splenogonadal fusion with atrophic testis, so simple excision of mass was done. This highlights the importance of laparoscopic assessment for the nature of the mass, which saves the patient from undergoing a radical surgery.

\section{Conclusion}

Splenogonadal fusion is a very uncommon disorder and due to its presentation as an asymptomatic testicular mass it can easily be misdiagnosed as malignancy though involved structure is of normal characteristics which suggests its benign nature. It must be considered as an important differential diagnosis of testicular masses, more so if it is associated with congenital anomalies. Preoperative laparoscopic assessment may prevent unnecessary radical orchidectomy but, in cases in which salvage is done for cryptorchidism, surveillance for detection of any future occurrence of malignancy is recommended.

\section{Ethical Approval}

Written informed consent to publication was obtained from the patient or next of kin.

\section{Conflict of Interests}

The authors declare that there is no conflict of interests regarding the publication of this paper.

\section{Acknowledgment}

The authors thank the patient for allowing them to publish the case report and use the images taken during his stay in hospital.

\section{References}

[1] A. M. Carragher, "One hundred years of splenogonadal fusion," Urology, vol. 35, no. 6, pp. 471-475, 1990.

[2] W. G. Putschar and W. C. Manion, "Splenicgonadal fusion," The American Journal of Pathology, vol. 32, no. 1, pp. 15-33, 1956.

[3] P. J. Le Roux and R. M. Heddle, "Splenogonadal fusion: is the accepted classification system accurate?" British Journal of Urology International, vol. 85, no. 1, pp. 114-115, 2000.

[4] W. A. Sneath, "An apparent third testicle consisting of a scrotal spleen," Journal of Anatomy \& Physiology, vol. 47, p. 340, 1913.

[5] D. Cortes, J. M. Thorup, and J. Visfeldt, "The pathogenesis of cryptorchidism and splenogonadal fusion: a new hypothesis," British Journal of Urology, vol. 77, pp. 285-290, 1996.

[6] A. B. M. Khairat and A. M. Ismail, "Splenogonadal fusion: case presentation and literature review," Journal of Pediatric Surgery, vol. 40, no. 8, pp. 1357-1360, 2005.

[7] R. Fernandez Atuan, P. Burgues Prades, A. Gonzalez Esgueda et al., "Splenogonadal fusion: case report and review of literature," Journal of Pediatric Surgery Case Reports, vol. 10, no. 1, pp. 375377, 2013.

[8] A. P. Steinmetz, A. Rappoport, G. Nikolov et al., "Splenogonadal fusion diagnosed by spleen scintigraphy," Journal of Nuclear Medicine, vol. 38, pp. 1153-1155, 1997.

[9] M. I. Karaman and E. T. Gonzales, "Splenogonadal fusion: report of 2 cases and review of the literature," Journal of Urology, vol. 155, pp. 309-311, 1996. 


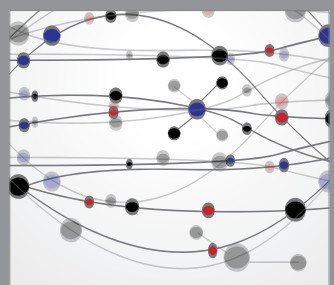

The Scientific World Journal
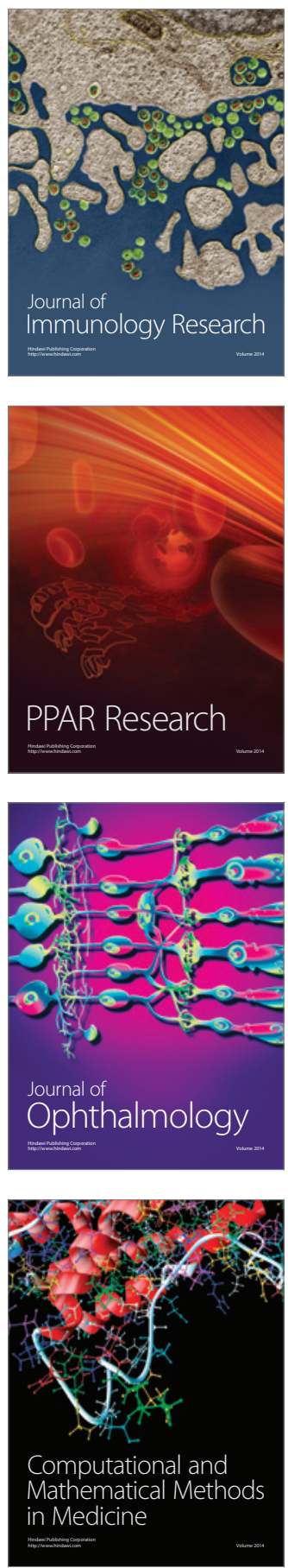

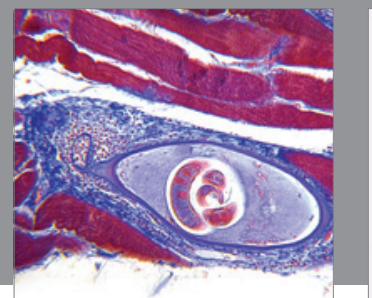

Gastroenterology

Research and Practice
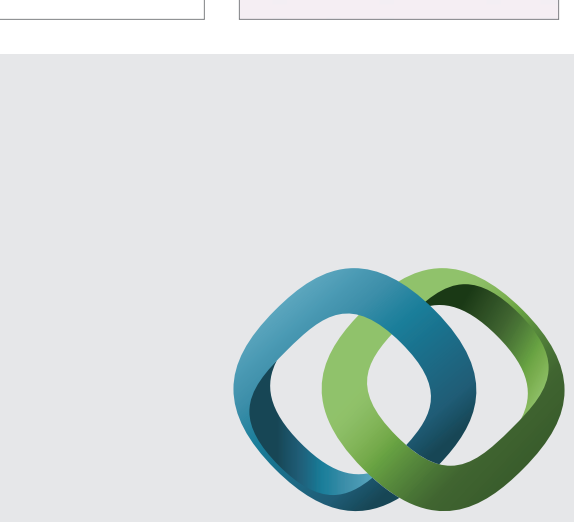

\section{Hindawi}

Submit your manuscripts at

http://www.hindawi.com
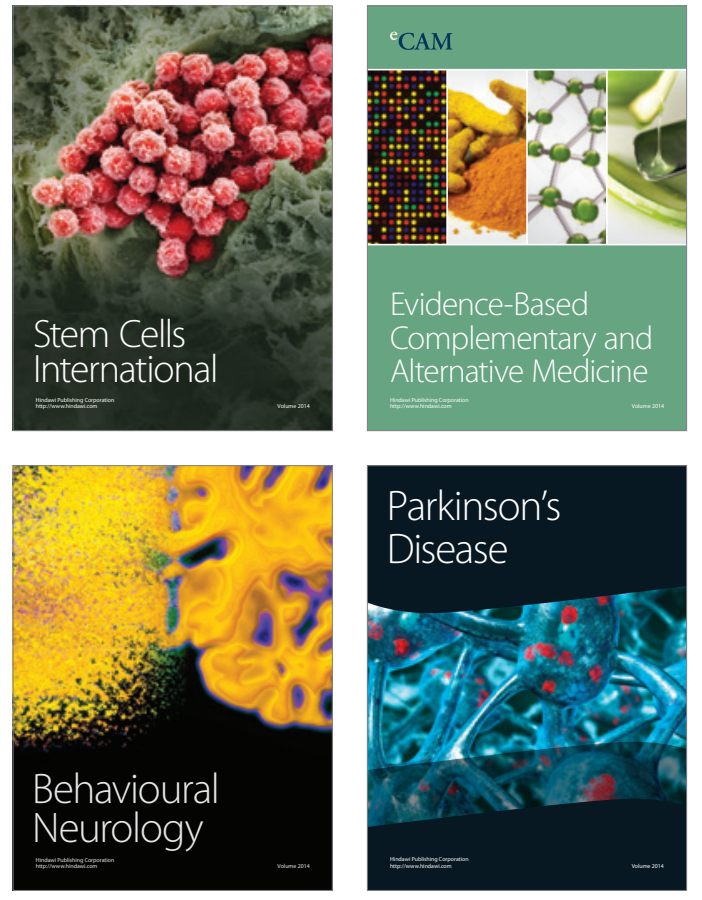
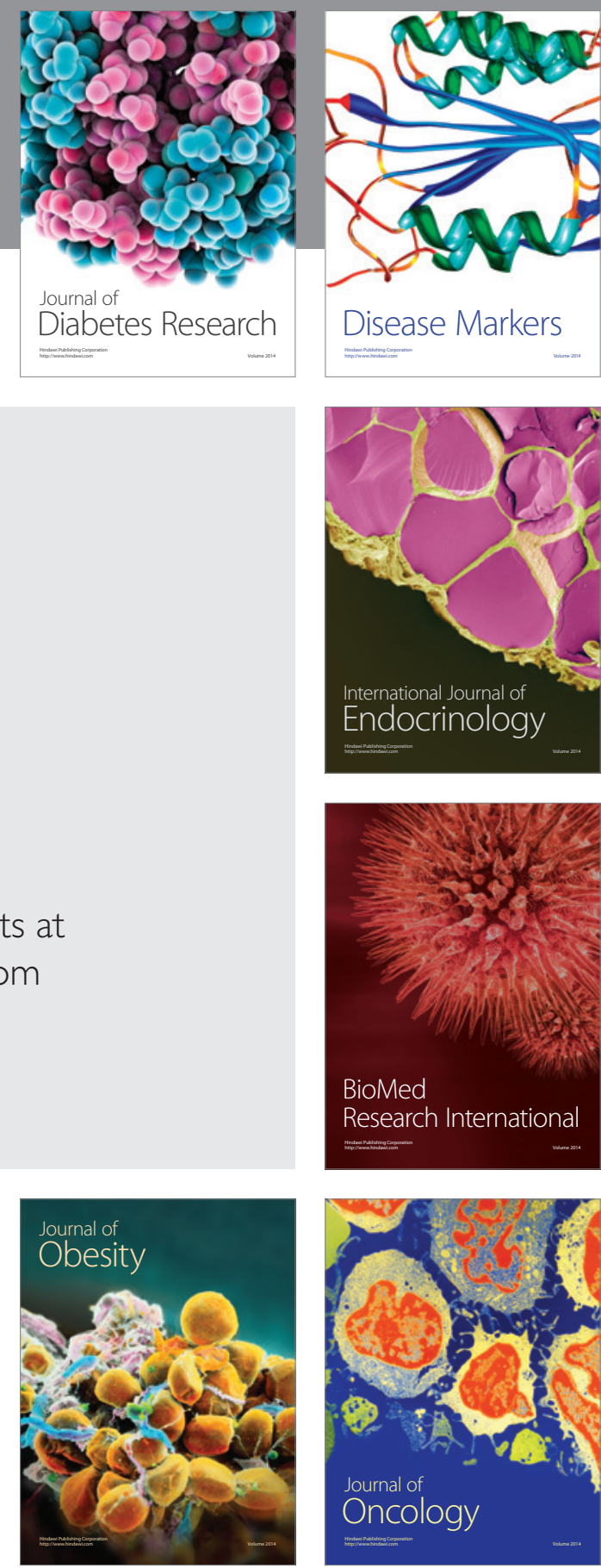

Disease Markers
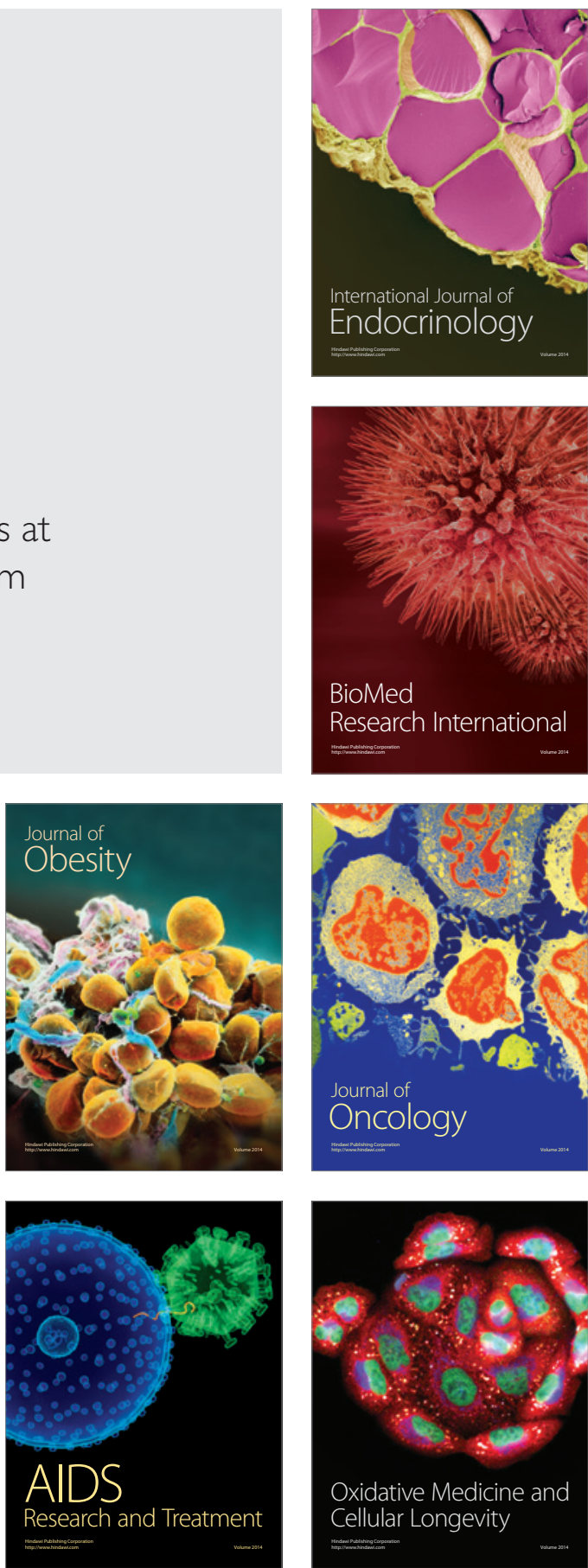